\title{
JOINTLY OPTIMIZED QUANTIZATION AND TIME DELAY ESTIMATION FOR SENSOR NETWORKS
}

\author{
Lavanya Vasudevan, Antonio Ortega, and Urbashi Mitra \\ University of Southern California \\ Department of Electrical Engineering \\ Los Angeles, California 90089-2564
}

\begin{abstract}
Sensor networks have emerged as a fundamentally new tool for monitoring inaccessible environments. Strict limitations on system bandwidth and sensor energy resources motivate the use of data compression at each sensor. Localization of unknown sources is an key application of sensor networks, requiring as an initial step, estimation of the time delay between signals received at different sensors. In this work, joint designs for quantizer-time delay estimator structures are presented. The goal for these new application-specific encoders/estimators is to achieve the best time delay estimate at a given bandwidth budget or latency bound, or minimize the rate required to reach an estimate with desired accuracy. For white sources, the optimal structure is shown to be a maximum-likelihood detector coupled with a maximum mutual information quantizer. Variations of this system are also considered: sequential detection schemes, empirical methods for unknown signal models, and rateconstrained methods. The proposed designs offer gains over those based on classical compression criteria.
\end{abstract}

\section{INTRODUCTION}

Sensor networks consist of a large number of low-power nodes cooperating to achieve a sensing goal. Typically, noisy measurements are collected from each sensor, and fused at some central site or node, to estimate an environmental parameter. These sensors are limited in power, memory, computational abilities and bandwidth. Computation is in general cheaper than communication [3], suggesting the use of data compression at the sensors. In this paper, we seek to develop algorithms to support collaboration and target localization in a sensor network; thus we consider time-delay

This research has been funded in part by the Integrated Media Systems Center, a National Science Foundation Engineering Research Center, Cooperative Agreement No. EEC-9529152; the Pratt \& Whitney Institute for Collaborative Engineering (PWICE) at USC; and the National Science Foundation Small ITR, CCR-0313392. Any opinions, findings and conclusions or recommendations expressed in this material are those of the authors and do not necessarily refiect those of the National Science Foundation. Early versions of this work were published in [1,2]. estimation, which is integral to these tasks. Thus, we consider systems where quantized data is sent from each of two sensors, and the fusion center estimates the associated time-delay based on received decoded data. Our goal is to design scalar quantization methods at the sensor which maximize the accuracy of the time-delay estimation, rather than simply reproduce the sensor data with some fidelity, e.g., a desired MSE level. We first cast time-delay estimation as a discrete, multi-hypothesis testing problem, develop the minimum probability of time delay estimator for our system model, and then design a novel scalar quantization scheme that optimizes the probability of error for this detector. We provide designs based on exact knowledge of source and noise statistics, and show that our processing-aware design outperforms standard detection-quantization schemes by achieving the best time delay estimate at a given latency bound, or minimizing the rate required to reach a required accuracy. We also present empirical techniques to train the quantizers and detectors on real data samples, and entropyconstrained approaches to operate within a rate budget.

Prior work $[4,5,6]$ on hypothesis testing with quantized data has focused on binary decisions in a NeymanPearson framework. This problem set-up does not translate directly to our multiple hypothesis, time-delay estimation problem. Since exact calculation of probability of error is generally intractable, these papers focus instead on optimizing asymptotic distance measures between the distributions under each of the two hypotheses (e.g. Kullback-Leibler distance, Chernoff bound). In more recent work [7, 8], the asymptotic bound on classification (multi-hypothesis testing) error probability is shown to be related to the smallest pairwise distributional distance. In our work, we explicitly derive a quantizer based on the probability of error. Interestingly, for our particular model, since all incorrect hypotheses are identically distributed, the resulting optimal quantizer maximizes the relative entropy (a distributional distance) between correct and incorrect hypotheses.

Standard scalar quantization seeks to encode the data from a source, characterized by its probability density function ( $p d f)$, with the lowest possible rate, and the smallest 
average distortion. The most common distortion measure is the mean squared error (MSE) between quantized and unquantized data [9]. Non-MSE based quantization for crosscorrelation detection includes the study in [10] of transform based quantization to optimize the Cramer-Rao bound for time-delay estimation. The transform based method comes at a cost of an increase in complexity and processing delays; furthermore, the correlator output depends on the reproduction levels of the quantizer, whereas our minimum probability of error detector is reproduction level independent. Further, instead of optimizing on a bound (CRB), which may or may not be achieved by a practical estimator, our novel quantizer directly optimizes the probability of error for the time-delay estimation task. In earlier work [11], we have presented quantizer designs for cross-correlation that minimize the squared error between quantized and unquantized correlation functions. Again, this is only an approximation to the probability of error, which should be (and is, in our new approach) the criterion for optimization.

\section{QUANTIZER AND DETECTOR DESIGN}

\subsection{Problem Formulation}

In this work, we study the special case of the two-sensor time-delay estimation subsystem. With multiple sensors, delay estimates can be obtained for pairs of sensors, and triangulated on sensor locations to determine the source position. Consider two sensors, capturing delayed and noisy versions of the same discrete signal, $x(\cdot)$ :

$$
\begin{aligned}
& x_{1}(m)=x(m)+w_{1}(m) \\
& x_{2}(m)=x(m+D)+w_{2}(m)
\end{aligned}
$$

The source process $X$, and noises $W_{1}, W_{2}$, are modeled as mutually independent, zero-mean, white processes, with known distributions. The unknown random delay $D$ is an integer with uniform probability mass over the set $\left[-D_{\max }, D\right.$ Each sensor processes its readings with a scalar quantizer. The quantizer is defined by an encoding function, $\alpha$, which maps quantizer inputs to discrete integer indices (or encoder bins), and a decoding function, $\beta$, which in turn maps the discrete indices into real-valued decoded outputs (or reconstructions). Let $\hat{x}_{i}(m)=\alpha\left(x_{i}(m)\right), i \in\{1,2\}$. We wish to estimate the time delay between two $M$-sample frames. Our goal is to design a quantizer-detector such that a time-delay estimate based on $\hat{x}_{i}(m)$ is as close as possible to $D$.

\subsection{Optimal Detector Design}

A simple estimate of $D$ is to determine the lag at which the unbiased quantized sample cross-correlation peaks:

$$
\tilde{D}=\arg \max _{n} \frac{1}{(M-n)} \sum_{m=1}^{M-n} \beta\left(\hat{x}_{1}(n+m)\right) \beta\left(\hat{x}_{2}(m)\right)
$$

The correlator detector is not necessarily optimal in the case of coarsely quantized sources. Further, solving for a quantizer that minimizes error probability for this detector is difficult, in particular because the result depends on both $\alpha(\cdot)$ and $\beta(\cdot)$ (see [11] for an approximation to this optimal quantizer). Thus, we pursue a joint design of quantizer and detector for our specific signal model, that exploits the discrete nature of the quantized data, and in particular does not require a decoder $\beta(\cdot)$ to be used. When $D$ is discretevalued, time-delay estimation becomes a multiple hypothesis testing problem with $2 D_{\max }+1$ choices. We propose the maximum a posteriori (MAP) test, which minimizes the probability of error for uniform misclassification costs.

Let hypothesis $H_{n}$ correspond to $n$ being the true delay between the two sequences. Let $Z$ denote the complete set of quantizer indices received by the central detector: $Z=\left\{\hat{x}_{1}(1), \cdots, \hat{x}_{1}(M), \hat{x}_{2}(1), \cdots, \hat{x}_{2}(M)\right\}$. For equally likely $H_{n}$, the MAP test is equivalent to the maximum likelihood (ML) test:

$$
\tilde{D}=\arg \max _{n} \operatorname{Pr}\left\{Z \mid H_{n}\right\}
$$

We wish to find the lag at which the two sequences "match" best, in the sense of greatest likelihood. Under hypothesis $H_{n}$, we determine the likelihood of this configuration:

$$
\begin{aligned}
& \begin{array}{lllll}
\hat{x}_{1}(1) & \ldots & \hat{x}_{1}(M-n) & \ldots & \hat{x}_{1}(M)
\end{array} \\
& \begin{array}{ccccc} 
& & \mathbb{1} & \Uparrow & \mathbb{1} \\
\hat{x}_{2}(1) & \ldots & \hat{x}_{2}(n+1) & \ldots & \hat{x}_{2}(M)
\end{array}
\end{aligned}
$$

With memoryless quantizers operating on a white source, $\operatorname{Pr}\left\{Z \mid H_{n}\right\}$ factors into a product of probabilities of corresponding pairs of symbols under hypothesis $H_{n}$. If $\Phi$ denotes the marginal probability mass function ( $p m f)$ of the quantizer outputs, and $\Theta$ the joint pmfunder correspondence,

$$
\begin{aligned}
\max \log \operatorname{Pr}\left\{Z \mid H_{n}\right\}= & \sum_{i=1}^{n} \log \Phi\left(\hat{x}_{2}(i)\right)+\sum_{j=1}^{M-n} \log \Theta\left(\hat{x}_{1}(j), \hat{x}_{2}(j+n)\right) \\
& +\sum_{k=M-n+1}^{M} \log \Phi\left(\hat{x}_{1}(k)\right)
\end{aligned}
$$

For known noise levels and quantizer structures, these $p m f \mathrm{~s}$ can be pre-computed and stored in a look-up table at the detector (see [2] for details for the case of Gaussian source and noises); calculation of Eq. (3) involves simple look-ups and sums of data in a finite range.

\subsection{Optimal Quantizer Design}

We next design the compression scheme. Under the MAP test, $\operatorname{Pr}\{$ Detection Error $\}=$

$\sum_{n} \operatorname{Pr}\left\{H_{n}\right\} \operatorname{Pr}\left\{\log \operatorname{Pr}\left\{Z \mid H_{n}\right\}<\log \operatorname{Pr}\left\{Z \mid H_{m}\right\}, m \neq n \mid H_{n}\right\}$ 
Let $n$ be the true delay between the two sequences. Recall from (3) that the metric $\log \operatorname{Pr}\left\{Z \mid H_{n}\right\}$ is the sum of (log) probabilities of each corresponding pair of symbols under this hypothesis. Let $\left(\hat{x}_{1}, \hat{x}_{2}\right)$ correspond at lag $n$. The probability of occurrence of this symbol pair is then $\Theta\left(\hat{x}_{1}, \hat{x}_{2}\right)$. The contribution of this symbol pair to the overall log likelihood sum at lag $n$ (the correct lag) is $\log \left(\Theta\left(\hat{x}_{1}, \hat{x}_{2}\right)\right)$. At any lag other than $n$, the symbols in this pair are considered independent; hence their metric contribution is $\log \left(\Phi\left(\hat{x}_{1}\right) \Phi\left(\hat{x}_{2}\right)\right)$. For low detection error, we would like to maximize the difference, averaged over all possible symbol pairs, between these quantities. The expected value of this difference is

$$
\sum_{\hat{x}_{1}} \sum_{\hat{x}_{2}} \Theta\left(\hat{x}_{1}, \hat{x}_{2}\right) \log \left(\frac{\Theta\left(\hat{x}_{1}, \hat{x}_{2}\right)}{\Phi\left(\hat{x}_{1}\right) \Phi\left(\hat{x}_{2}\right)}\right)
$$

Eq. (4) is the relative entropy between the joint distributions at match and mismatch, or the mutual information between the two quantized sequences at the correct delay. We call the quantizer which maximizes Eq. (4), the MaxMI quantizer.

\section{EMPIRICAL QUANTIZER DESIGN}

Empirical quantizer designs, based on unquantized "training" samples that are typical of sensor data, are useful when a design using closed form models becomes complex or impractical (e.g., for a large number of quantization levels) or when models are not readily available (e.g., for vector quantization, or unknown source and noise statistics). We introduce an entropy constrained design that explicitly takes into account the rate in empirical quantizer design.

Assume that we have access to two length- $M$ noisy input sequences $z_{1}$ and $z_{2}$ such that $z_{2}(m)=z_{1}\left(m+D_{t}\right)+$ $\epsilon\left(m+D_{t}\right)$, where $\epsilon\left(m+D_{t}\right)$ is an unknown noise term and $D_{t}$ the known true delay. These sequences could be obtained through direct measurements on an environment of interest. Our objective is to design a quantizer which maximizes the entropy-constrained empirical mutual information between the quantized sequences $\hat{z}_{1}$ and $\hat{z}_{2}$, when they are aligned at $D_{t}$.

Algorithm:

1. Initialize the iteration number $k=0$. Choose an initial $N$-level quantizer $Q_{0}$, say uniform. Initialize the empirical mutual information to $\tilde{I}_{0}=0$. Align the two sequences at $D_{t}$. Let $\alpha_{k}$ and $\beta_{k}$ denote the encoder and decoder mappings, respectively, at the $k$-th iteration.

2. Quantize all input signals: $\alpha_{k}\left(z_{i}\right)=\hat{z}_{i}, i=\{1,2\}$.

3. Find empirical symbol probabilities $\tilde{\Phi}[i]$ and joint probabilities $\tilde{\Theta}[i, j]$, by computing the frequency of occurrence of the corresponding encoder output symbols $i$ and symbol pairs $(i, j)$. Assign the reproduction levels to be the centroids of each quantization bin, i.e., $\beta_{k}(i)=\mathbf{E}\left[z \mid \alpha_{k}(z)=i\right]$.

4. Determine the empirical mutual information: $\tilde{I}_{k}=$ $\sum_{i=1}^{N} \sum_{j=1}^{N} \tilde{\Theta}[i, j] \log \frac{\tilde{\Theta}[i, j]}{\tilde{\Phi}[i] \tilde{\Phi}[j]}$; and the empirical rate $\tilde{R}_{k}=\sum_{i=1}^{N} \tilde{\Phi}[i] \log \frac{1}{\tilde{\Phi}[j]}$. If $\exp \left\{-\tilde{I}_{k}\right\}+\lambda_{R} \tilde{R}_{k} \geq$ $\exp \left\{\tilde{I}_{k-1}\right\}+\lambda_{R} \tilde{R}_{k-1}$ terminate procedure'

5. Consider the two input sequences pair by pair. For the entire frame, re-assign quantizer indices to input samples in the following manner: For an input sample pair $\left(z_{1}(m), z_{2}(m)\right)$, pick that assignment $(i, j)$ which minimizes this cost function:

$$
\begin{aligned}
D(i, j)= & \exp \left\{-\tilde{\Theta}[i, j] \log \frac{\tilde{\Theta}[i, j]}{\tilde{\Phi}[i] \tilde{\Phi}[j]}\right\} \\
& +\lambda \sqrt{\left(z_{1}(m)-\beta_{k}(i)\right)^{2}+\left(z_{2}(m)-\beta_{k}(j)\right)^{2}} \\
& -\lambda_{R}(\tilde{\Phi}[i] \log \tilde{\Phi}[i]+\tilde{\Phi}[j] \log \tilde{\Phi}[j])
\end{aligned}
$$

The first term encourages picking that (vector) assignment which would add most to the overall mutual information. However, this does not guarantee that the resulting quantizer will be regular ${ }^{2}$; The second term, weighted by Lagrange factor $\lambda_{N N}$, is used to penalize assignments which cause input samples to stray too far from the closest quantization bin. The third term, weighted by Lagrange factor $\lambda_{R}$, is used to encourage assignments with low rate contribution. $\lambda_{R}=0$ would be a design with no rate constraint.

6. Determine the new empirical symbol probabilities $\tilde{\Phi}[i]$ generated by this re-assignment. To reinforce regularity, we solve for the regular quantizer $\alpha_{k}$ with boundaries such that the resulting bin probabilities match the empirical symbol probabilities $\tilde{\Phi}[i]$.

7. Increment iteration counter: $k=k+1$; go to (2).

\section{SIMULATIONS AND RESULTS}

Computer simulations were run with randomly generated i.i.d. Gaussian and Gauss-Markov sources and i.i.d. Gaussian noise signals, both mean-zero. SNR was measured as the ratio of source variance to noise variance. Scalar quantization codebooks and partitions were generated based on a training set for the MSE and Empirical MaxMI quantizers, and designed on the joint $p d f$ of source and noise for the

\footnotetext{
${ }^{1}$ Since $\exp \{-\tilde{I}\}+\lambda_{R} \tilde{R}$ is a non-monotonic function of the quantization bin sizes, we look for the point at which it changes direction from increasing to decreasing: this is the function maximum.

${ }^{2}$ Maximizing MI along might force $\beta\left(\alpha\left(z_{i}(a)\right)\right)>\beta\left(\alpha\left(z_{i}(b)\right)\right)$, for samples such that $z_{i}(a)<z_{i}(b)$.
} 
model-based MaxMI quantizer. The 2-level quantizer was assigned reproduction levels \pm 1 , as in [12]. Noise was independently added to two copies of the source signals; the true delay between them was set to a randomly generated integer between -10 and 10 ; and they were quantized with each of the three encoders. The number of detection errors were averaged over 0.1 to 1 million iterations. Block lengths ranged from 30 through 105 , SNR's from $+7 \mathrm{~dB}$ to $-1 \mathrm{~dB}$. We plot the probability of error associated with sample frames of varying sizes. The block length would translate with suitable scaling into bit rate, when the quantizer outputs are encoded into bits. Thus, error probability can be traded off against processing delay or transmission cost.

Fig. 1 presents model-based block detection simulation results for i.i.d. Gaussian data. In all cases, the combination of MAP detector and MaxMI quantizer provides best performance. Note that the 2-level quantizer is trivially the MaxMI quantizer for 2 levels; we observe that while the 3and 4-level quantizers have higher entropy, they achieve a required level of accuracy with fewer samples. In following sections, we demonstrate that our proposed methods can be optimized to meet both total rate and error probability targets, yielding an entropy-constrained design.

The multiple hypothesis testing framework in Section 2.2 can be applied in a straightforward manner to a sequential detector, such as the Multi-hypothesis Sequential Probability Ratio Test (MSPRT) algorithm described in [13]. Data is received sample-by-sample; the likelihood of the current set of data under each hypothesis is compared against a threshold based on the desired error rate. As soon as one of these likelihoods exceeds the threshold, the corresponding hypothesis is declared winner and reception stops. The authors show that for a fixed target error probability, the expected sample size needed to make a decision is inversely proportional to the distributional distance. Since the MaxMI quantizer maximizes this distance, it is optimal for this detector (Fig. 2).

In Figs. 3(a) and 3(b) we show results of the empirical design for quantizers for larger number of bins (e.g., 8) for which model-based solutions are intractable. Figs 3(a) shows an 8-level empirical quantizer (without entropy constraints) applied to i.i.d. Gaussian data at low SNR, and 3(b) shows a 6-level entropy-constrained empirical quantizer applied to i.i.d. Gaussian data at moderate SNR. For a sufficient number of quantization bins our combined quantizer/detector can outperform correlation based techniques that use unquantized data. Performance can be traded off with transmitted rate under the entropy-constrained design.

\section{CONCLUSIONS AND FUTURE WORK}

In this paper we have presented a novel, application-specific, joint detector-quantizer design for time delay estimation in sensor networks. We pursue non-standard quantization designs where we jointly optimize the quantizer and the timedelay estimator (a multi-hypothesis detector). This approach results in a quantizer which maximizes the mutual information between correctly and incorrectly aligned quantized signals. Our jointly optimized scheme outperforms standard mean-squared-error based quantization coupled with a correlator-based time-delay estimator. Further, the optimal maximum likelihood detector for quantized signals is of lower complexity than the correlator scheme. We have successfully generalized our model-based methods to realworld scenarios with empirical and rate-constrained methods. Future work will include studies of optimal designs for correlated data. Tracking, non-homogeneous sensors and multiple sensor problems are also of interest.

\section{REFERENCES}

[1] L.Vasudevan, A.Ortega, and U.Mitra, "Applicationoptimized quantizers for time-delay estimation in sensor networks," in Proc. of 2003 Workshop on Statistical Signal Proc., St. Louis, MO, Sept.-Oct. 2003.

[2] L.Vasudevan, A.Ortega, and U.Mitra, "Applicationspecific compression for time delay estimation in sensor networks," in ACM Sensys '03, Los Angeles, CA, November 2003.

[3] G.J.Pottie and W.J.Kaiser, "Wireless integrated network sensors," Communications of the ACM, vol. 43, no. 5, May 2000.

[4] H.V.Poor and J.B.Thomas, "Applications of AliSilvey distance measures in the design of generalized quantizers for binary decision systems," IEEE Transactions on Communications, vol. 25, no. 9, September 1977.

[5] T.J.Flynn and R.M.Gray, "Encoding of correlated observations," IEEE Transactions on Information Theory, vol. 33, no. 6, November 1987.

[6] M.Longo, T.D.Lookabaugh, and R.M.Gray, "Quantization for decentralized hypothesis testing under communication constraints," IEEE Transactions on Information Theory, vol. 36, no. 2, March 1990.

[7] A.Jain, P.Moulin, M.I.Miller, and K.Ramchandran, "Information-theoretic bounds on target recognition performance based on degraded image data," IEEE Transactions on Pattern Matching and Machine Intelligence, vol. 24, no. 9, September 2002.

[8] T.S.Han and S.Amari, "Statistical inference under multi-terminal data compression," IEEE Transactions on Information Theory, vol. 44, no. 6, October 1998. 


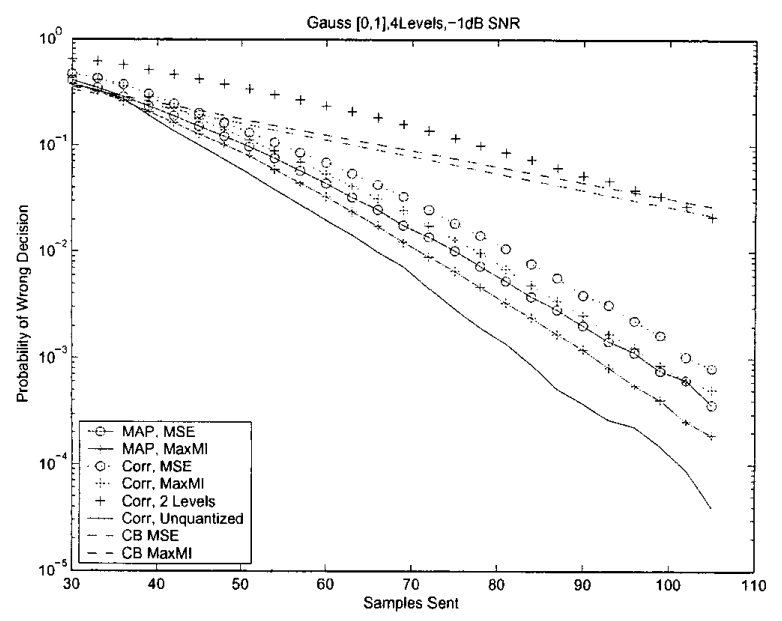

Fig. 1. Model-based detector-quantizer designs described in Sections 2.3 and 2.2, applied to i.i.d. Gaussian data.

[9] A.Gersho and R.M.Gray, Vector Quantization and Signal Compression, Kluwer Academic Press, 1991.

[10] M.Chen and M.L.Fowler, "Optimizing non-MSE distortion for data compression in emitter location systems," in 2003 Conference on Information Sciences and Systems. The Johns Hopkins University, March 2003.

[11] L.Vasudevan and A.Ortega, "Processing-aware compression for sensor networks," in 2nd IEEE Sensor Array and Multichannel Signal Processing Workshop, August 2002.

[12] H.Wang, D.Estrin, and L.Girod, "Preprocessing in a tiered sensor network for habitat monitoring," EURASIP JASP Special Issue On Sensor Networks, March 2003.

[13] C.W.Baum and V.V.Veeravalli, "A sequential procedure for multihypothesis testing," IEEE Transactions on Information Theory, vol. 40, no. 6, November 1994.

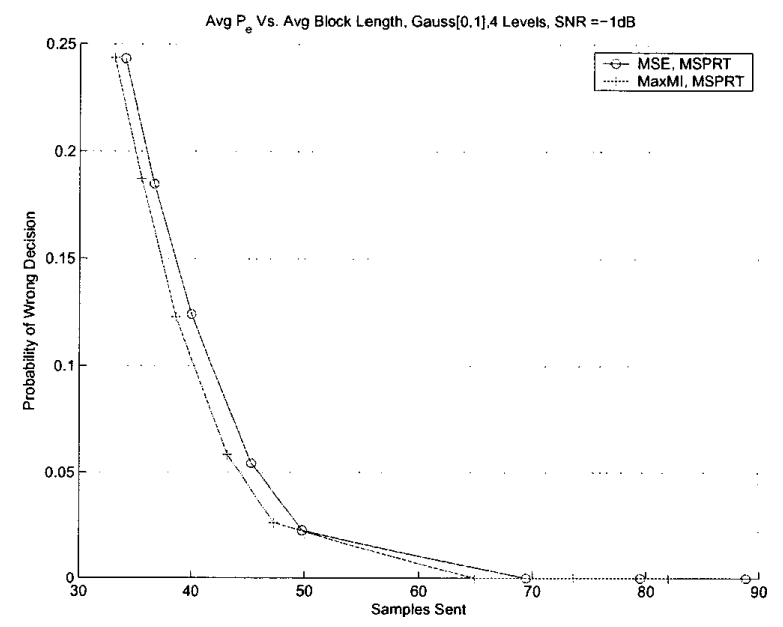

Fig. 2. Sequential MAP detector (MSPRT, [13]) applied to i.i.d. Gaussian data. 


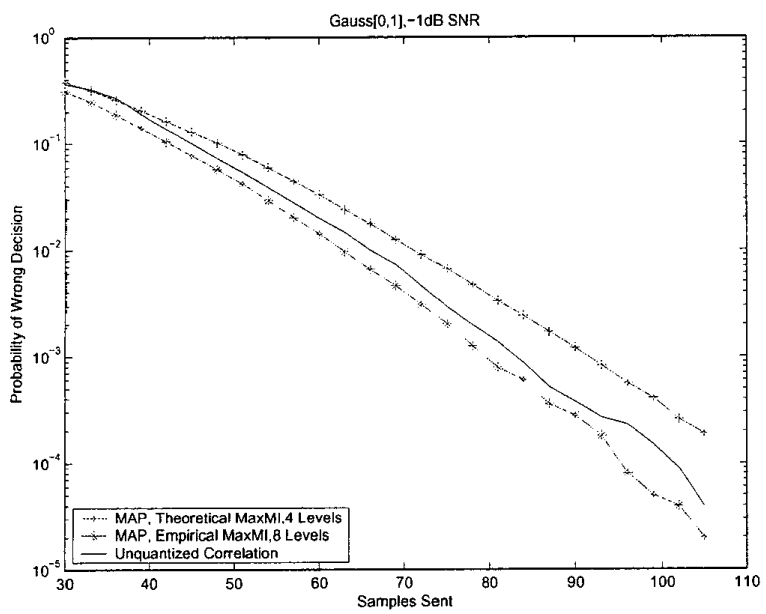

(a) Without Entropy Constraint, Low SNR

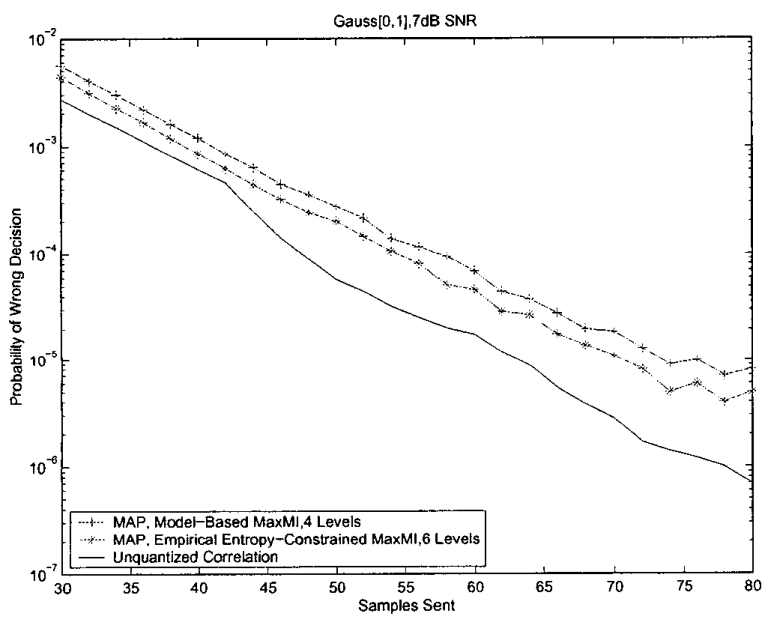

(b) With Entropy Constraint, Moderate SNR

Fig. 3. Empirical quantizer design algorithms described in Section 3, applied to i.i.d. Gaussian data. 